\title{
LARGE STRUCTURES: WHICH SOLUTIONS FOR HEALTH MONITORING?
}

\author{
Géraldine Camp ${ }^{\mathrm{a}}$, Pierre Carreaud ${ }^{\mathrm{b}}$, Hervé Lançon ${ }^{\mathrm{c}}$
}

${ }^{a}$ Metrology Instrumentation Survey Department Manager, SITES, Rueil-Malmaison, France, geraldine.camp@ sites.fr ${ }^{\mathrm{b}}$ Surveying, Laser and Photogrammetry Project Manager, SITES, Rueil-Malmaison, France, pierre.carreaud@sites.fr

${ }^{\mathrm{c}}$ Technical Director, SITES, Ecully, France, herve.lancon@sites.fr

KEY WORDS: Large structures, dams, viaducts, cooling towers, tunnels, monitoring, instrumentation, geometric survey, 3D laserscanning, photogrammetry, deformation, degradation, mapping, technology

\begin{abstract}
:
Whatever the age of a large structure (dam, viaduct, cooling tower, nuclear containment, tunnel,...) It has to be periodically monitored. It is a challenge to realise these services when the access is limited and difficult for Man. This paper introduces a global approach, developed by SITES, through examples of application on different concrete dams or cooling towers, and their results. This global method involves three techniques: the SCANSITES ${ }^{\circledR}$ (a visual inspection system), the LIDAR (3D laser scanning) and high resolution photogrammetry.
\end{abstract}

\section{INTRODUCTION}

Lots of Large structures like dams, towers, tunnels, were built in the 60's. Lots of them are still here and working 60 years later. Some of these industrial projects might be categorised in the next decades as historical heritage for their rareness, their innovation or their size.

It would be catastrophic if these structures were to fail, as they have too great an importance: economic or emblematic significance, or just for safety reasons. In this way and regarding their size and constitution, which are exceptional, the structural health of dams, cooling towers, viaducts, or nuclear containment has to be monitored in order to avoid heavy and expensive repairs that might be done too late and using methods which would require shutdown of the facility.

For decades, among the existing monitoring devices and methodologies applied to different types of large structures, two are widely used for safety management: visual inspection and geometric survey. The first is usually carried out with empiric methods, and the second is realized using accurate but discrete methods such as geodetic micro-triangulation or sensors instrumentation.

This paper introduces a new approach, using an exhaustive and numeric method called SCANSITES $3 \mathrm{D}^{\circledR}$.

The SCANSITES $3 \mathrm{D}^{\circledR}$ is based on the combination of the SCANSITES $^{\circledR}$ method, an advanced tool which provides numeric defect inspection of large structures, a new wide ranged LIDAR technology aiming to deliver 3D exhaustive geometric mapping, and very high resolution photogrammetric coverage. In the first part of this paper, we will introduce the SCANSITES $^{\circledR}$ method, the LIDAR coverage and the photogrammetry. In the second part, we will explain how the combination is achieved and which data can be extracted on large structures. Before concluding, we will extend this paper to tunnel auscultation.

\section{SCANSITES ${ }^{\circledR}$ OVERVIEW}

Due to concrete or metal material use, knowledge of the structure cracking, corrosion and visual evolution is necessary for monitoring.
In the past, many owners weren't completely satisfied with the traditional defect mapping process, using binoculars or rope access. The main drawback is the difficulty to produce a scaled defects map enabling an accurate and reproducible monitoring (crack evolution, opening measurement...). To answer this problem, the SCANSITES ${ }^{\circledR}$ was developed in the 1990's. This system aims to produce a digital defects mapping connected to a database which is working as a true real-time G.I.S. (Geographic Information System) "Figures 1 and 2". It is composed of:

- a hardware tool with a robotized video inspection head (focal length from 200 to more than $4000 \mathrm{~mm}$ ) and its controllers "Figure 1",

- a software suite including a database and several dedicated inspection tools (defects localization, cracks opening measurement, geometric and pathological characteristics, mapping tools) "Figure 2".

The whole system is designed to operate on-site, without heavy carriage. Several dozens of dams, cooling towers and nuclear containment building, viaducts and chimneys have been examined by the SCANSITES ${ }^{\circledR}$, in France and across the world, by SITES Company team.

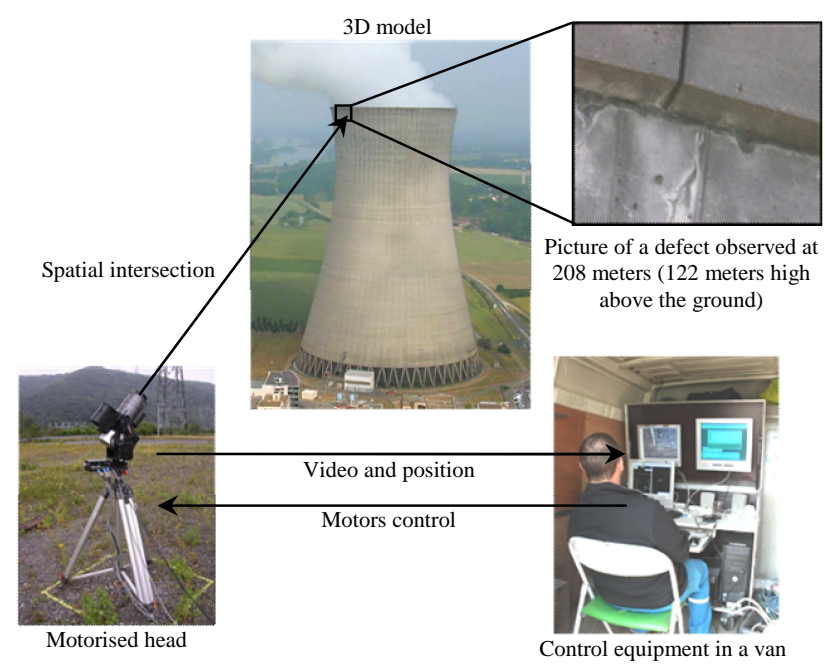

Figure 1. SCANSITES ${ }^{\circledR}$ in operation 


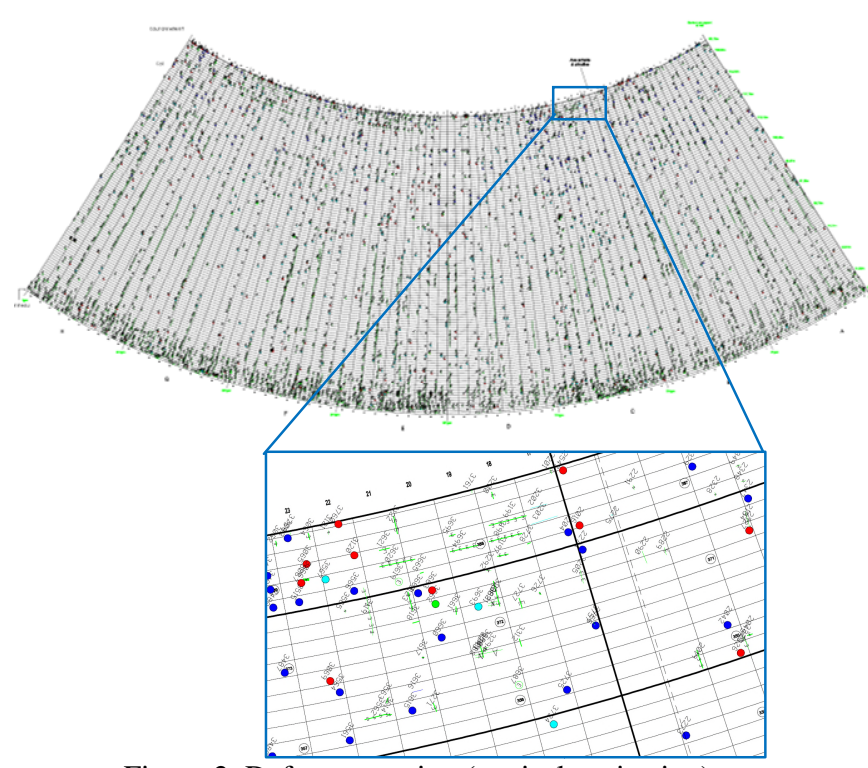

Figure 2. Defects mapping (conical projection)

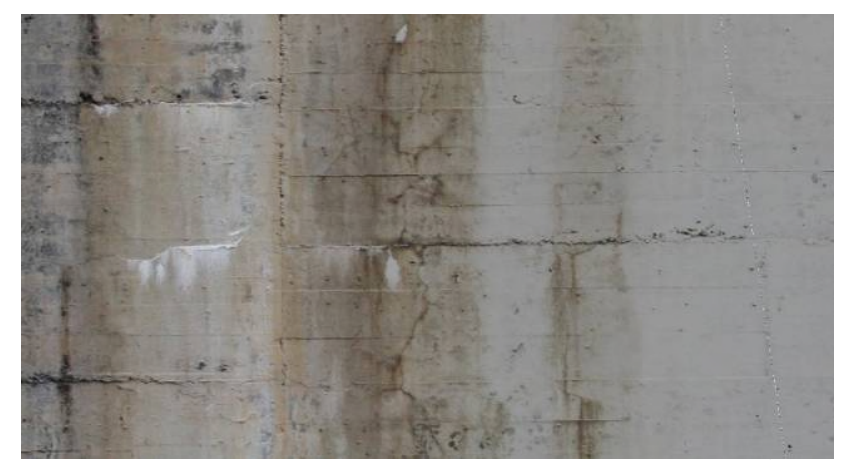

Figure 3. Picture of a defect captured with SCANSITES ${ }^{\circledR}$

\section{LIDAR OVERVIEW}

The LIDAR "Figure 4" is a device which produces high density surveying in 3D coordinates. This technology is mature and has been used for long range applications for less than 10 years.

It is based on two angular coders linked to mirrors (horizontal and vertical) and a remote laser distance measurement device (time of flight of laser emission or phase shift technology). The system works with enough velocity to acquire more than 100000 points each second. For the majority of large structures, a wide range LIDAR is used. It is able to scan structures, up to 1000 meters onto surfaces.

The result of a LIDAR survey, called "point cloud" "Figure 5". It is composed of dozen of millions points known in XYZ and laser intensity. The average density is 1 point each 5 to $20 \mathrm{~mm}$ and the accuracy of the modelling surface is a few millimetres (5-10 mm). The surface can be compared to theorical shapes in order to extract the deviations.

Most of the time, 3 to 5 points of view are needed for a dam, 6 to 8 for cooling towers. The different point clouds have to be linked to each other and placed into a single coordinate system based on the gravity.

LIDAR is not limited to large structures. Nowadays, short range units with full dome $360^{\circ}$ field of view are available in order to scan and archive the geometry of castles, monuments and other inside purpose. Some of these units can catch up to 1 million of points in 1 second with max range of 100 meters. It is used in SITES company for tunnel inspection.

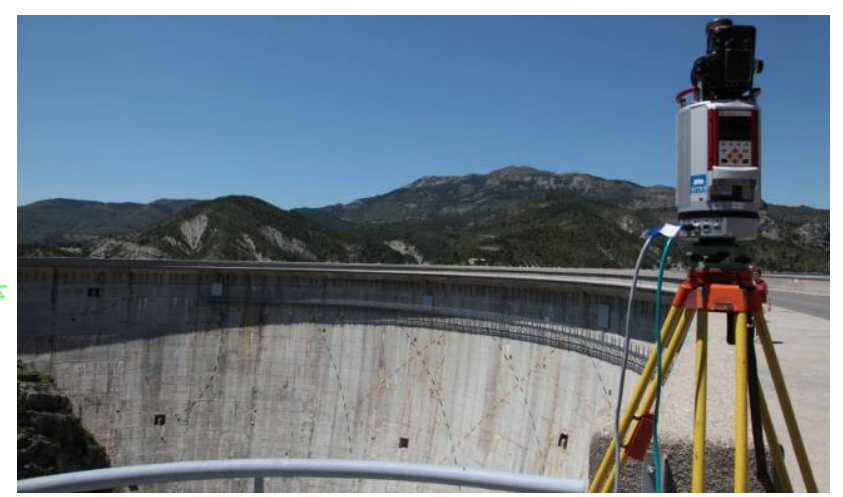

Figure 4. LIDAR in operation on the left bank of a dam

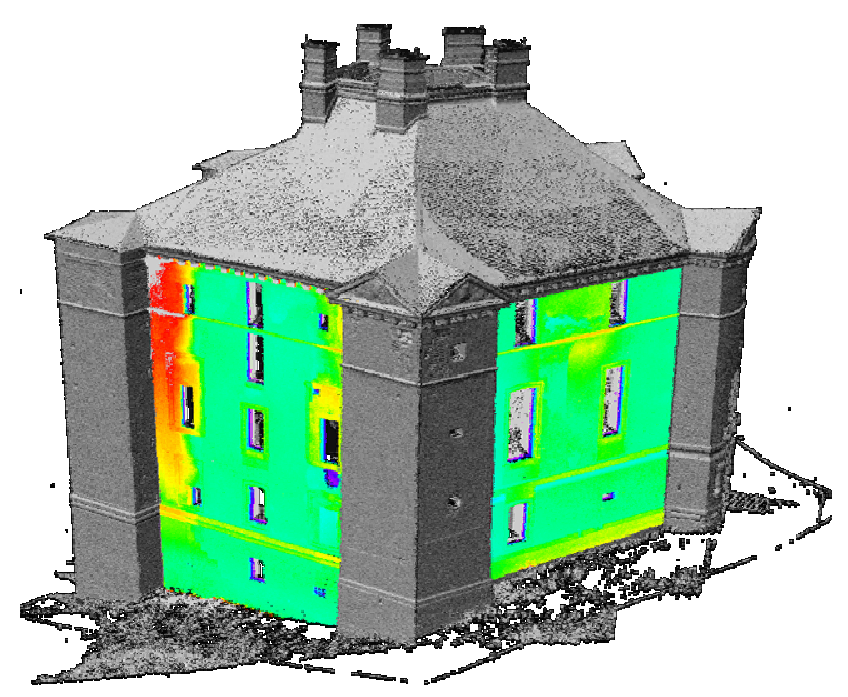

Figure 5. Point cloud of Castle - deviation to mean plane

\section{HIGH DEFINITION ORTHOPHOTOGRAPHIC COVERAGE}

The photogrammetric coverage delivers exhaustive and high definition pictures of the structure, including the parts without any defects.

It is possible to produce a visual inspection, using a long focal lens and high definition digital camera in order to produce referenced pictures. "Figure 6" The camera and lenses used can give a pixel equivalent to a few millimetres onto the structure, which makes it possible to detect the main defects.

As the photos' positions and orientations are known (XYZ and Euler angle), each photo can be projected on the 3D mesh to texture it. The next step is to project the textured 3D mesh on a primitive (plane, cone, and cylinder) to obtain a map. This projected assembly of image is called orthophotography. "Figure 7"

At each point of view, complete photo coverage is done with a given focal lens: more than 1000 photos can be done for one point of view which represents about 10000 photos for the complete detailed inspection of a cooling tower.

After the projection and assembly of each photo, the size of the orthophotography can reach up to 25 billion of pixels.

More and more, with the digital photography and computing progress, the inspection based on orthophotography can completely replace the SCANSITES ${ }^{\circledR}$ inspection. The field operations and associated treatments were designed in order to be fully compatible and replaceable: the tools used to do the inspection, database, mapping and results are the same. 


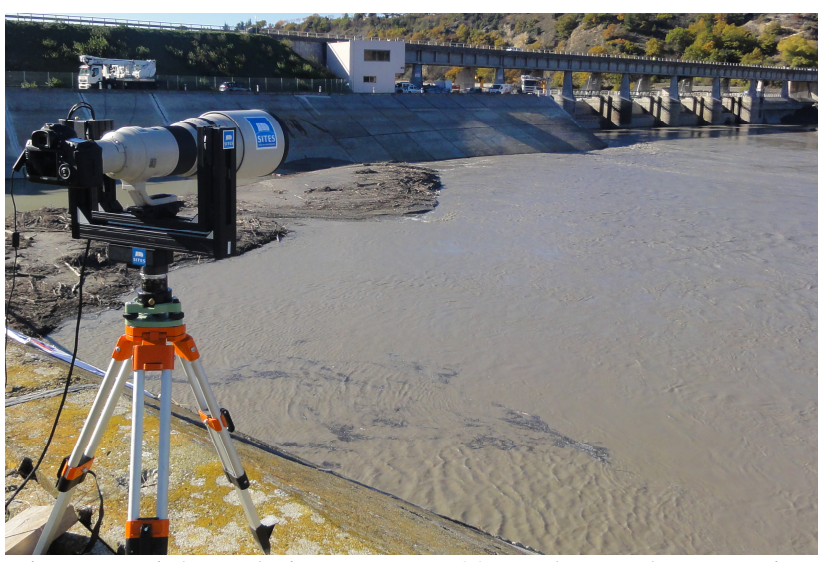

Figure 6. High resolution camera, $600 \mathrm{~mm}$ lens and panoramic head facing upstream wall of a dam

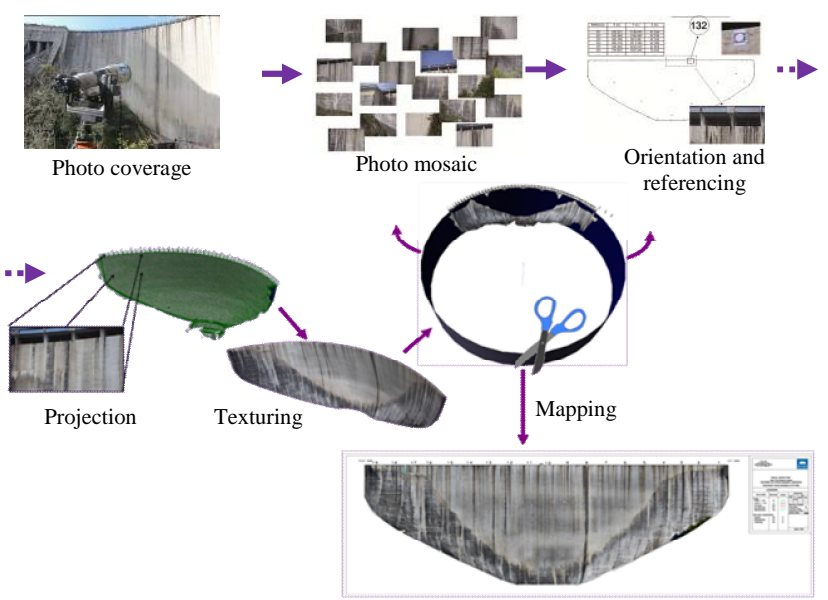

Figure 7. Block diagram of orthophotography operations

\section{OPERATIONS}

The SCANSITES $3 \mathrm{D}^{\circledR}$ monitoring requires that all data is known in a single $\mathrm{XYZ}$ referential. The method can use the one established for the traditional survey (targets, pillars, geodetic points). In case there is no available network, it is necessary to create one, based on singular points on the structure and referenced with traditional survey operations or laser scanning (LIDAR).

Regarding the visual inspection, each structure owner has its own requirements. It deals with defects, which have to be surveyed, and the associated classification. One of the most important parameters is the minimum opening for a crack that needs to be surveyed. It mainly impacts the focal length used during the inspection (up to 4000 millimetres) and the total number of defects stored. All those considerations help in preparing the mission, mainly the database and the inspection software.

The LIDAR, the SCANSITES ${ }^{\circledR}$ and/or the photogrammetric head are set at different locations in order to cover the structure's entire surface. The high gain video camera and quality lenses of the SCANSITES® allow it to work with low ambient luminosity. The LIDAR, for its part, can work without light.

With the LIDAR, a complete scan is realized. Based on this point cloud, a triangular meshing "Figure 8" is generated and converted in a 3D shape. The first use of this 3D shape is to allow the SCANSITES ${ }^{\circledR}$ to locate the defects in 3D and/or to project the photos in order to create the very high resolution orthophotography.

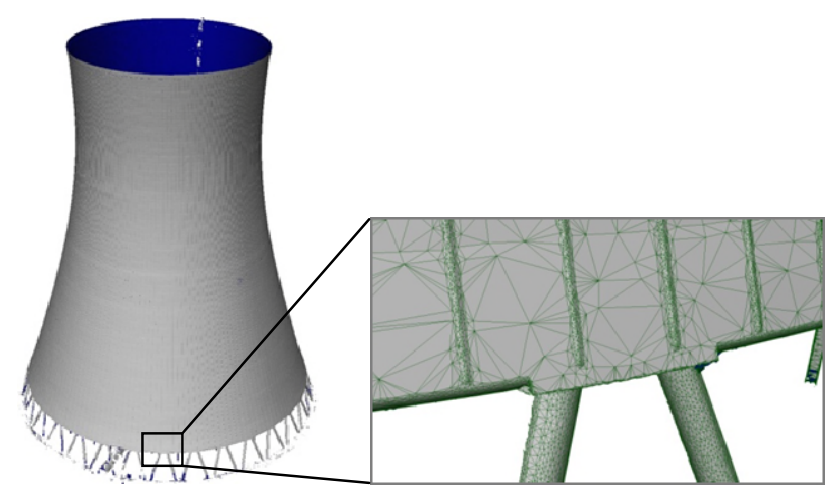

Figure 8. Triangular meshing

With this incoming data, the visual inspection is processed. With the SCANSITES ${ }^{\circledR}$, the operator inspects the entire wall by moving the inspection head with a joystick. When a defect is found, it is photographed. The 3D map is updated in real time with defects and the database is filled with its characteristics and coordinates. In parallel or in replacement of this operation, a complete high definition photogrammetric coverage is done with a high resolution camera and a robotised panoramic head. The operations done to inspect the orthophotography use the same software than the real time SCANSITES ${ }^{\circledR}$ tool but this inspection is realized at the office.

\section{TREATMENTS}

The treatments aim to produce, on a multilayer file, a map containing all defects captured, the geometric deflections and the photogrammetric coverage.

The first step is to compare the structure's 3D shape to the theoretical shape or to a previous survey. The 3D deflections are extracted and a map is generated. "Figures 10, 12" Two ways of representation are possible. One is a coloured map: each colour depends on the deflection value. The other way is to carry out a contour line representation.

The second step is to overlay the defects surveyed with the SCANSITES $^{\circledR}$ or orthophotography, using the same referential network.

The last step is to overlay the pictures directly on the structure's $3 \mathrm{D}$ shape enabling the production of an orthophotography. With these files, many views can be generated such as composite views: defects/deflections, defects/pictures, or thematic views (based on database queries).

\subsection{Case study: a concrete arch dam in France}

The SCANSITES3D ${ }^{\circledR}$ method was applied to a large dam located in France. Its main figures are a height of 95 meters and a crest length of 200 meters. The average distance between the points of view and the downstream facing was about 100 meters. The aim of this job was to connect the geometric deflections to the defects surveyed. The LIDAR survey recorded 70 million points, and the defects' total quantity was near 4000. "Figures 9, 10"

The focal lens of photogrammetric coverage has been adjusted between 100 and $500 \mathrm{~mm}$ in order to produce an orthophotography of 1.5 billion pixels with a pixel size of $5 \mathrm{~mm}$. The detailed inspection was performed by the SCANSITES ${ }^{\circledR}$ tool with a focal length of $1000 \mathrm{~mm}$.

The defects and photogrammetric coverage have been mapped using a cylindrical projection based on the mean cylinder of the downstream wall.

This monitoring was helpful to locate the parts to repair and to store the visual and geometric aspect of the wall. 


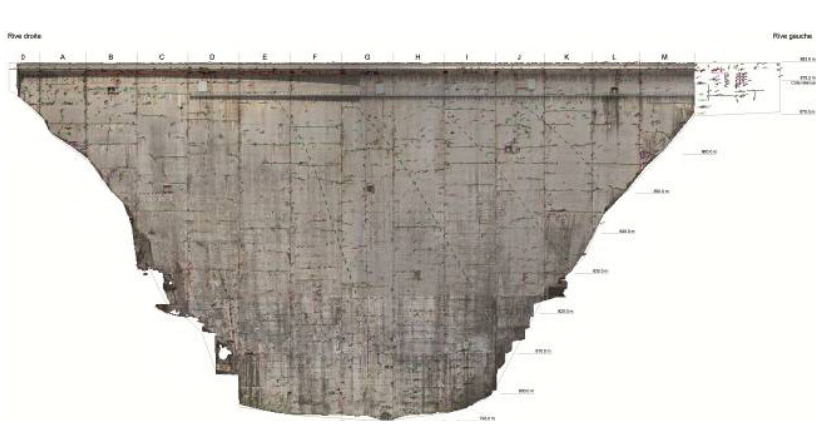

Figure 9. Orthophotography of the downstream wall with defects overlaid

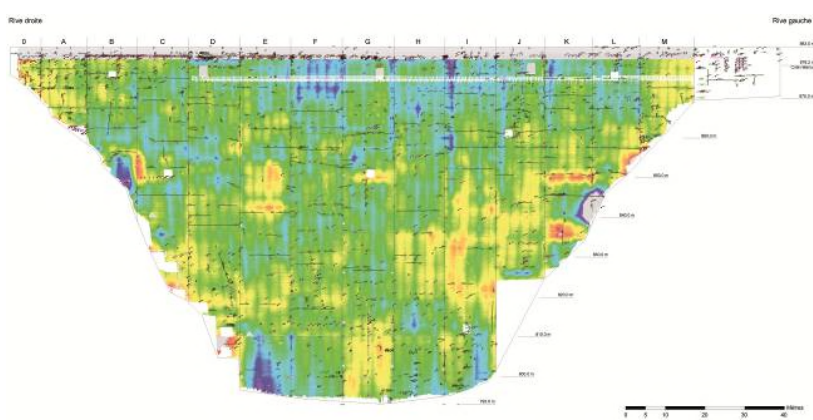

Figure 10. Map overlaying deflection/defects and magnifying

\subsection{Case study: a concrete cooling tower in France}

The second case study concerns a cooling tower located in Europe. Its dimensions are a height of 90 meters and a diameter of 50 meters at the bottom.

The aim of the job was to get the tower's geometric deflection and an accurate visual inspection with a global photogrammetric coverage "Figures 11 and 12".

As carried out on the previous case study (dam), a LIDAR point cloud was generated representing 150 million points. In parallel, we covered the whole shell with high resolution pictures, projected it on a $3 \mathrm{D}$ shape, and then on a projection cone, for a total quantity exceeding 25 billion pixels.

In order to generate the orthophotography, several processors and computers were used in order to reduce the projection time. Without the parallel computing, the treatment time would be more than two weeks.

On this type of structure, mostly built in the 70's and 80's, we've observed that the real shape can be far from the theoretical shape.

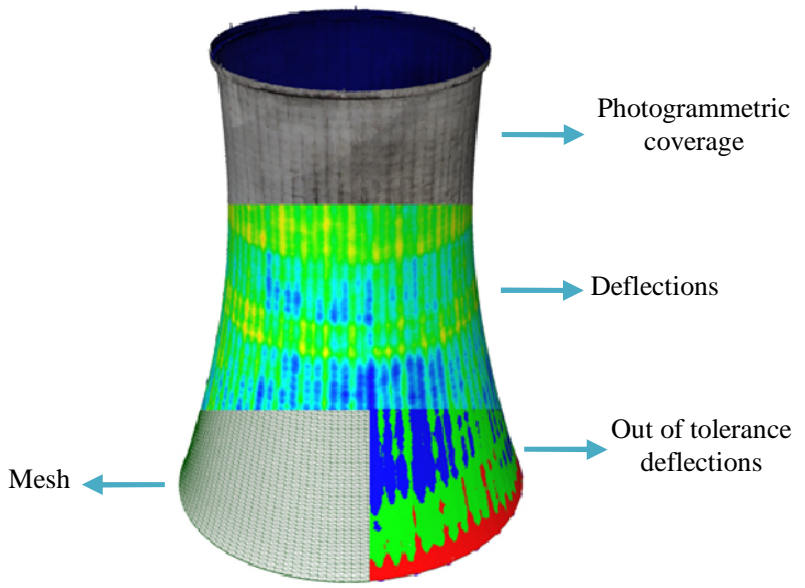

Figure 11. Representation of 3D model, deflections and photogrammetric coverage

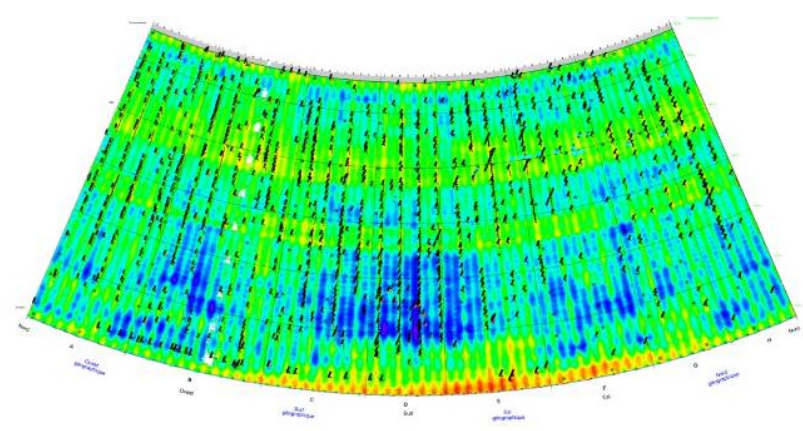

Figure 12. Map overlaying deflection/defects and magnifying

\section{RESULTS}

\subsection{Statistics}

Concerning the visual inspection, the traditional way is to produce a sketch based on a close inspection (rope access) or binocular observations. The consequent difficulties of these methods are to get a good location of the defects and their evolution. SCANSITES3D ${ }^{\circledR}$ provides some digital results: a scaled defect map and defects database. The first result is to produce an accurate report emphasizing defects evolution between two inspections, and the number of defects divided by zone "Figure 13".

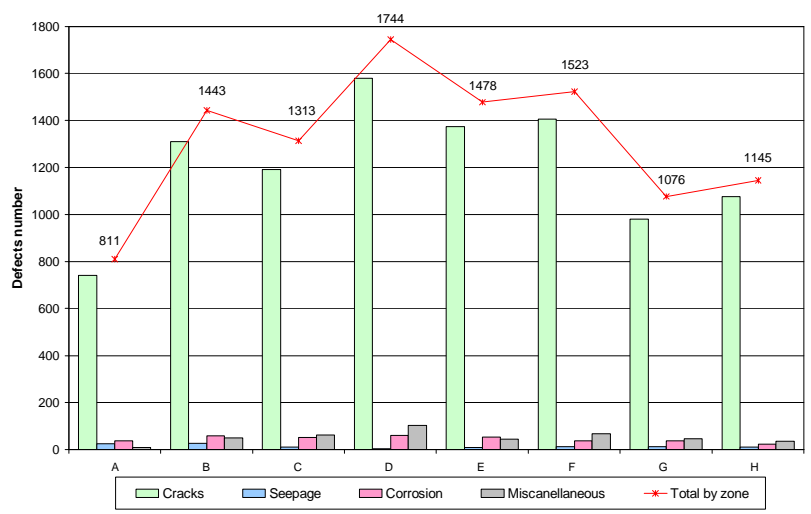

Figure 13. Number of defects by family and zone

The LIDAR coverage is a guideline for defects analysis, for instance to see if cracks are correlated, or not, with geometrical distortions.

Another interesting point is its use on parts covered by moss. Scanning one way we get structural information where visual inspection is inefficient. It is also helpful to know where a structural diagnosis has to focus on (with concrete sample or testing core for example).

\subsection{Use of the data for repairing and utility of geometry}

The data extracted from the visual inspection is useful to establish an accurate bill of quantities for restoration works, like total crack length to be treated, total corroded bar amount for passivation treatment...

Providing the exact length and the exact position of cracks and corrosions allows the preparation of a reparation intervention with all the necessary information: quantity of product to use, localisation (height from the floor or the crest,...) and simulations for the access (length of rope and inclination, height of positive or negative crane, ...)

As the accurate 3D shape of the structure is known, data for planning sensor installation or well location can be easily computed. 


\subsection{Advantage of LIDAR compared to traditional survey}

Traditional geometric survey uses theodolites and well-known micro-triangulation methods. It has the advantage of providing results close to the best possible accuracy $(1$ to $10 \mathrm{~mm})$. However, the drawback is that it is a "discrete" method, since it focuses on a limited number of points, usually a few dozen (target, reflectors), not necessarily placed on critical parts.

Even if SCANSITES3D ${ }^{\circledR}$ is less accurate, the high density scan produces some surface definitions close to a 3-4 $\mathrm{mm}$ uncertainty, and we get global continuous information. It greatly improves the sensitivity of the geometric diagnosis, showing all the details.

In addition, the geometrical surveys with LIDAR were much quicker to carry out than the traditional geodesic method.

Another advantage is that this method works on every structure, even if there is no surveying equipment such as targets.

\subsection{GIS (Geographic Information System) consultation}

All the data (photo, geometric survey, defects maps and evolutions) is overlaid on a same file. The engineer gets a faster way to make his diagnosis compared to the fastidious data fusion imposed by separate reports.

The last advantage is the storage of all collected data in a database, offering efficient tools to measure the structure's ageing

All these jobs are performed without rope access, improving dramatically the safety conditions.

\section{EXTENSION TO TUNNEL AUSCULTATION}

A version of the SCANSITES3D ${ }^{\circledR}$ has been adapted for tunnel auscultation. The tools are about the same: short range laser scanner, digital camera with wide lens, thermographic camera and odometer mounted on a trolley. The data is mapped on a mean cylinder representing tens of kilometers of tunnel wall enabling direct access to the visual aspect, distortions linked and position in the tunnel (kilometer point - KP).

The view rendered and referenced is useful to detect leaks, concretions and parts of the concrete ready to fall. "Figures 14, 15 and $16 "$.

The resulting file size can reach tens of billions of pixels, depending on the length of the tunnel... Each part of the tunnel can be viewed with a computer: visual aspect, geometry. This helps the owner for repairation and new equipment installation as the maintenance access is very restricted to a few hours during the night or weekend for high traffic tunnels.
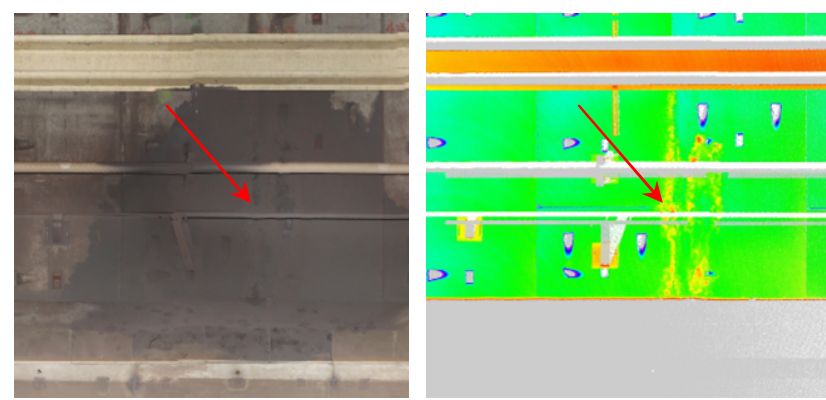

Figure 14. Water leakage and concretions (left : orthophoto - Right : distortion map)

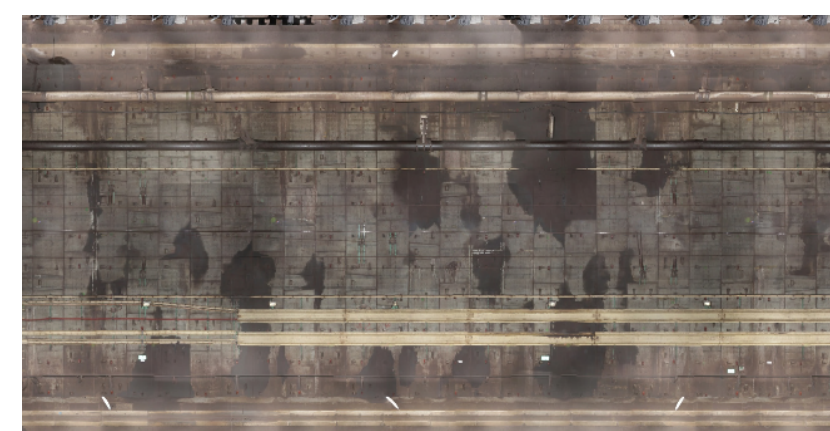

Figure 15. Orthophotography of Tunnel (cylindrical projection)

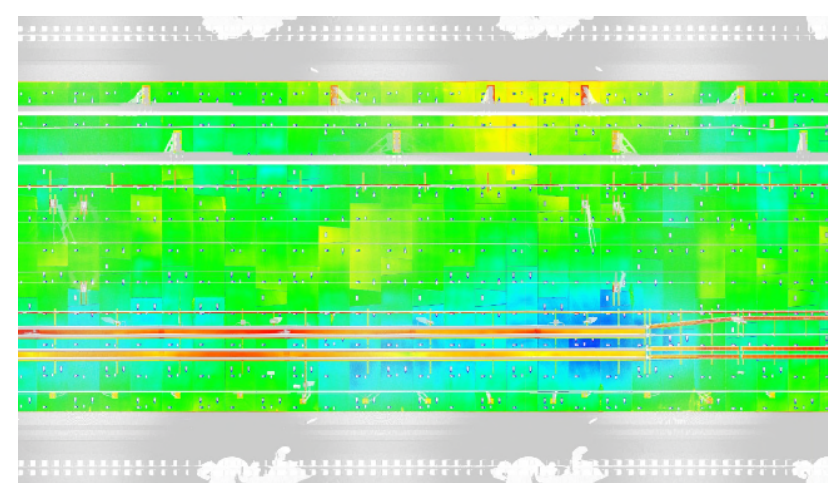

Figure 16. Distortions of Tunnel (cylindrical projection)

\section{CONCLUSION}

We've presented a new and modern approach for visual inspection and geometric survey, here focused on dams and cooling towers, with SCANSITES3D ${ }^{\circledR}$. This method is particularly convenient for every structure which needs the resuming of its monitoring program, because it provides an exhaustive inventory. It also allows the readjustment of an existing monitoring program by completing the partial survey done by conventional approaches. Not only is this method adapted to concrete structures, but it can also be used on old constructions, masonry-works, clay works... Finally, the correlation between defects and deflections is precious information used to locate the areas where geodic surveying and sensors have to focus on. Moreover, besides useful results for the monitoring, the SCANSITES3D ${ }^{\circledR}$ provides as-existing mappings which are often lacking on old structures. This method is widely applicable on large structures such as dams, but also skyscrapers, chimneys, cooling towers, pillars of viaducts, tunnels, ...

The next step, in progress, is to overlay a high accuracy thermographic imagery survey. Its aim is to study the possible gain in diagnosis, mainly on cracks. 